\title{
THE DISCRETE NATURE OF THE PALEY-WIENER SPACES
}

\author{
CAROLYN EOFF
}

(Communicated by Albert Baernstein II)

\begin{abstract}
The Shannon Sampling Theorem suggests that a function with bandwidth $\pi$ is in some way determined by its samples at the integers. In this work we make this idea precise for the functions in the Paley-Wiener space $E^{p}$. For $p>1$, we make a modest contribution, but the basic result is implicit in the classical work of Plancherel and Pólya (1937). For $0<p \leq 1$, we combine old and new results to arrive at a characterization of $E^{p}$ via the discrete Hilbert transform. This indicates that for such entire functions to belong to $L_{p}(\mathbf{R}, d x)$, not only is a certain rate of decay required, but also a certain subtle oscillation.
\end{abstract}

\section{INTRODUCTION}

In this paper we study, for $0<p$, the space $E_{\tau}^{p}$ of entire functions $f$ of finite exponential type $\tau$ for which

$$
\|f\|_{p}^{p}=\int_{-\infty}^{+\infty}|f(x)|^{p} d x<+\infty .
$$

$E_{\tau}^{p}$ is clearly a subspace of $L_{p}(\mathbf{R}, d x)$, so \|\|$_{p}$ is a norm for $1 \leq p$ and a quasinorm for $0<p<1$. Recall that an entire function $f$ is of exponential type $\tau$ if $f(z)=\mathscr{O}\left(e^{((\tau+\varepsilon)|z|)}\right)$ for all $\varepsilon>0$.

For the sequel, we essentially consider $\tau=\pi$, as the other cases are handled by a change of variables. Henceforth, $E_{\pi}^{p}=E^{p}$. Our definition of $E^{p}$ is motivated by a classical theorem of Paley and Wiener.

Theorem 1 (Paley and Wiener). For an entire function $f$ to belong to $E^{2}$, it is necessary and sufficient that there exist $\psi \in L_{2}([-\pi, \pi])$ such that

$$
f(z)=\int_{-\pi}^{\pi} \psi(t) e^{i t z} d t .
$$

Basic facts about entire functions can be found in [1]; in particular, for $f$ in $E^{p},|f(x)| \rightarrow 0$, as $|x| \rightarrow+\infty$. This allows for the observation that, unlike the $L_{p}(\mathbf{R}, d x)$ spaces, the $E^{p}$ spaces are nested: $E^{p} \subseteq E^{q}$, if $0<p \leq q$.

Received by the editors January 14, 1993 and, in revised form, May 19, 1993; presented by the author at the AMS Special Session on Holomorphic Spaces, II, Joint Meeting of the AMS-MAA, San Antonio, Texas, January 13, 1993.

1991 Mathematics Subject Classification. Primary 30D10, 30D55.

This work was supported in part by National Science Foundation grant NSF-DMS-9008763. 
Many facts about $E^{p}, 0<p<2$, follow from known facts about $E^{2} .\left(E^{2}\right.$ is denoted $P W$ by some authors, e.g., [7].)

A brief review of some of these facts: $E^{2}$ is the isometric image of $L_{2}([-\pi, \pi])$ under the inverse Fourier transform and is therefore a Hilbert space. Generally speaking, a function whose Fourier transform is supported in an interval is said to be band-limited; such functions are interpreted as signals, with no frequencies outside the "band". $E^{2}$ seems to play a significant role in signal processing applications [5]. Central to the $E^{2}$ theory is the so-called sinc function

$$
\operatorname{sinc}(z)=\frac{\sin \pi z}{\pi z}
$$

Since $\operatorname{sinc}(z-n)$ is the image of $e^{-i n t} / \sqrt{2 \pi}$ under the inverse transform, the collection $\{\operatorname{sinc}(z-n)\}_{n \in Z}$ is an orthonormal basis of $E^{2}$.

The cardinal series of a function $f$ is

$$
f(x)=\sum_{n=-\infty}^{+\infty} f(n) \operatorname{sinc}(x-n) .
$$

Many facts about the history of the cardinal series and especially its place in communication theory can be found in the comprehensive article of $\mathrm{J}$. R. Higgins [5]. As we will see, for $p>1$ the sinc functions play the same role as the standard unit vectors in $l_{p}$. Although the sinc functions do not belong to $E^{p}$, for $0<p \leq 1$, they are still central to our results. A bit of notation: In this paper, $l_{p}$ will denote the space of $p$-summable sequences indexed on the integers. Also, the term samples of a function $f$ will always refer to the sequence $\{f(n)\}_{n \in \mathbf{Z}}$.

\section{2. $E^{p}$ IS A QUASI-BANACH SPACE}

Although $E^{p}$ is clearly a subspace of $L_{p}(\mathbf{R}, d x)$, it does not seem to have been noticed that $E^{p}$ is complete, for values of $p$ other than 2 . To show that $E^{p}$ is closed, it suffices to prove that convergence in $E^{p}$ forces uniform convergence on compact subsets of $\mathbf{C}$ and preserves type. This and more will follow from the following results of Plancherel and Pólya [6].

Theorem 2 (Plancherel and Pólya). Let $p, \tau>0$ and $f \in E_{\tau}^{p}$.

(i) For $y \in \mathbf{R}$

$$
\int_{-\infty}^{+\infty}|f(x+i y)|^{p} d x \leq e^{p \tau|y|} \int_{-\infty}^{+\infty}|f(x)|^{p} d x
$$

(ii) There exists a constant $A>0$, which depends only on $\tau$ and $p$ so that

$$
\sum_{n=-\infty}^{+\infty}|f(n)|^{p} \leq A \int_{-\infty}^{+\infty}|f(x)|^{p} d x
$$

Let $z_{0}=x_{0}+i y_{0} \in \mathbf{C}$ and denote $f_{z_{0}}(u)=f\left(u+z_{0}\right)$. If $f \in E^{p}$, then $f_{z_{0}}$ 
also belongs to $E^{p}$. Applying (i) and (ii) we see that

$$
\begin{aligned}
\left|f\left(z_{0}\right)\right|^{p} & =\left|f_{z_{0}}(0)\right|^{p} \\
& \leq \sum_{-\infty}^{\infty}\left|f_{z_{0}}(k)\right|^{p} \\
& \leq B \int_{-\infty}^{\infty}\left|f_{z_{0}}(t)\right|^{p} d t \\
& =B \int_{-\infty}^{\infty}\left|f_{i y_{0}}\left(t+x_{0}\right)\right|^{p} d t \\
& =B \int_{-\infty}^{\infty}\left|f_{i y_{0}}(t)\right|^{p} d t \\
& =B \int_{-\infty}^{\infty}\left|f\left(t+i y_{0}\right)\right|^{p} d t \\
& \leq B e^{p \pi|y|} \int_{-\infty}^{\infty}|f(t)|^{p} d t .
\end{aligned}
$$

Consequently, for $f \in E^{p}$,

$$
\left|f\left(z_{0}\right)\right| \leq B e^{\pi|y|}\|f\|_{p}
$$

From this, we see that if $\left(f_{n}\right)$ is a Cauchy sequence in $E^{p}$, then it is Cauchy with respect to the topology of uniform convergence on compacta. Consequently, the limit function is not only in $L_{p}(\mathbf{R})$, but is entire and of exponential type $\pi$. These observations yield the following result.

Theorem 3. For $0<p, E^{p}$ is complete with respect to the \|\|$_{p}$ quasinorm.

At this point, perhaps it is worthwhile to list some of the bounded operators on $E^{p}$ which are of natural interest. It is obvious that real translation maps $E^{p}$ isometrically into itself, and it follows from Theorem 2 that complex translation also maps $E^{p}$ boundedly into itself. The map $f \rightarrow f_{c}$, where $f_{c}(z)=f(c z)$, is a bounded map into $E^{p}$ for $|c| \leq 1$, but in general $f_{c}$ may not belong to $E^{p}$, for $|c|>1$. Also, it follows from the work of Plancherel and Pólya in [6] that differentiation is a bounded operator from $E^{p}$ into itself. Finally, it should be observed that part (i) of Theorem 2 implies that the map $f \rightarrow e^{i \pi z} f$ is an isometry from $E^{p}$ into $H^{p}$ of the upper half-plane.

3. $E^{p}$ IS ISOMORPHIC TO $l_{p}, p>1$

At the heart of our subsequent results is the following classical result of Plancherel and Pólya [6].

Theorem 4 (Plancherel and Pólya). Let $p, \tau>0$ and let $f \in E_{\tau}^{p}$.

(i) If $\tau<\pi$, then there exists a constant $B>0$ which depends only on $\tau$ and $p$ so that

$$
\int_{-\infty}^{+\infty}|f(x)|^{p} d x \leq B \sum_{n=-\infty}^{+\infty}|f(m)|^{p} .
$$

(ii) If $\lim _{z \rightarrow \infty} f(z) e^{-\pi|z|}=0$ and if $1<p$, then (i) holds and the constant $B$ depends only on $p$. 
Now Plancherel and Pólya proved that if a function $f$ of exponential type $\tau$ also belongs to $L_{p}(\mathbf{R})$, then $f$ satisfies $\lim _{z \rightarrow \infty} f(z) e^{-\tau|z|}=0$. Theorems 2 and 4 allowed them to make the following observation.

Corollary 1. Let $1<p$. There exist constants $C_{1}$ and $C_{2}$ (depending only on $p)$ such that for all functions in $E^{p}$

$$
C_{1} \sum_{n=-\infty}^{+\infty}|f(n)|^{p} \leq \int_{-\infty}^{+\infty}|f(x)|^{p} d x \leq C_{2} \sum_{n=-\infty}^{+\infty}|f(n)|^{p} .
$$

Plancherel and Pólya used this fact to prove that for a function $f$ in $E^{p}$, $p>1$, the cardinal series

$$
\sum_{n=-\infty}^{\infty} f(n) \operatorname{sinc}(z-n)
$$

converges en moyenne d'ordre $p$ vers $f$ [6]. Conversely, it is clear from the corollary and Theorem 3 that given a sequence $\left\{\alpha_{n}\right\}$ in $l_{p}$ one can show that the resulting cardinal series

$$
F(z)=\sum_{n=-\infty}^{\infty} a_{n} \operatorname{sinc}(z-n)
$$

represents a unique function in $E^{p}$, with samples $\left\{\alpha_{n}\right\}$. It is also evident that the sinc functions form a basis, in fact, an unconditional basis for $E^{p}, p>1$. These remarks, together with Corollary 1 , yield the following result.

Theorem 5. Let $p>1$. E $E^{p}$ is isomorphic to $l_{p}$ via the mapping $f \rightarrow\{f(n)\}_{n \in \mathbf{Z}}$.

This result has an obvious consequence.

Corollary 2. For $p>1,\left(E^{p}\right)^{*} \cong E^{q}$, where $\frac{1}{p}+\frac{1}{q}=1$.

We see that the action of a linear functional $\phi \in\left(E^{p}\right)^{*}, \phi \sim g \in E^{q}$, is given by

$$
\phi(f)=\sum_{n \in \mathbf{Z}} f(n) \overline{g(n)}=\int_{-\infty}^{+\infty} f(x) \overline{g(x)} d x
$$

for all $f \in E^{p}$.

4. $E^{p}$ IS ISOMORPHIC TO THE DISCRETE HARDY SPACE $H^{p}(\mathbf{Z}), 0<p \leq 1$

In many situations, $p=1$ is a critical value. Recall from the Hardy space theory that since the Hilbert transform is bounded on $L_{p}, p>1, H^{p}$ and $L_{p}$ are essentially the same. However, for $0<p \leq 1, H^{p}$ turns out to be a proper closed subspace of $L_{p}$ consisting of functions which not only satisfy the size condition but also possess a certain type of cancellation. Indeed, $H^{p}$ consists of those functions in $L_{p}$ for which the Hilbert transform (viz., the conjugation operator) is bounded (e.g., see [4] or [2]).

We have found that a parallel situation exists for $E^{p}$ with respect to $l_{p}$. Now $l_{p}=L_{p}(\mathbf{Z}, d \sigma)$ for $\sigma=$ counting measure; so, by analogy with the standard theory (and at the risk of overusing the Hardy space notation and terminology), 
we define the discrete Hardy space, $H^{p}(\mathbf{Z}), 0<p<\infty$, to consist of those sequences $\alpha=\left\{\alpha_{k}\right\} \in l_{p}$ which satisfy

$$
\sum_{k \in \mathbf{Z}}\left|\sum_{n \neq k} \frac{\alpha_{n}}{k-n}\right|^{p}<+\infty .
$$

Thus $H^{p}(\mathbf{Z})$ is the subspace of $l_{p}$ consisting of those sequences $\alpha=\left\{\alpha_{n}\right\}$ for which the discrete Hilbert transform also belongs to $l_{p}$.

The discrete Hilbert transform, $H$, of a sequence $\alpha=\left\{\alpha_{n}\right\}$ is defined by

$$
H(\alpha)(k)=\sum_{n \neq k} \frac{\alpha_{n}}{k-n} .
$$

Note that for any noninteger $c \in \mathbf{R}, H_{c}(\alpha)(k)=\sum_{n \in \mathbf{Z}} \alpha_{n} /(k-n+c)$ yields the same class of sequences. $H_{c}(\alpha)$ is convolution of the sequence $\alpha$ with the kernel $1 /(n+c)$. For the sequel, we will use $H_{c}$ with $c=\frac{1}{2}$, which we will (by a small abuse of notation) denote by $H$.

$H^{1}(\mathbf{Z})$ is mentioned by Coifman and Weiss [2, p. 622] as an example of a Hardy space, $H^{p}(\mathbf{X})$, associated with a space $\mathbf{X}$ of homogeneous type; these spaces are the result of extending the atomic decomposition theory for the classical Hardy spaces to more general settings. A caveat regarding notation: $H^{p}(\mathbf{X})$ is defined atomically in [2]; thus it is not obvious that $H^{p}(\mathbf{Z})$ as defined above coincides with the corresponding atomic space of the same label in [2], although it is easily seen to contain the atomic space. (Coifman and Weiss suggest that the two are the same [2]; we shall consider the connection in a later paper.)

A priori, it would appear that, for $p>1, H^{p}(\mathbf{Z})$ and $l_{p}$ are different. However, Plancherel and Pólya [6] proved that if a sequence $\alpha=\left\{\alpha_{n}\right\} \in l_{p}$ for $p>1$, then there is a constant $C>0$, so that

$$
\|H(\alpha)\|_{l_{p}} \leq C\|\alpha\|_{l_{p}} .
$$

(A discrete version of the M. Riesz theorem.) Thus, for $p>1, H^{p}(\mathbf{Z})$ and $l_{p}$ coincide.

For $0<p \leq 1$, we define an obvious quasinorm on $H^{p}(\mathbf{Z})$. For $\alpha=$ $\left\{\alpha_{n}\right\}_{n \in \mathbf{Z}}$,

$$
\|\alpha\|_{H^{p}}=\|\alpha\|_{l_{p}}+\|H(\alpha)\|_{l_{p}} .
$$

That $H^{p}(\mathbf{Z})$ is complete with respect to this quasinorm will follow from subsequent results.

We recall Plancherel and Pólya's inequality for functions of type strictly less than $\pi$.

$$
\int_{-\infty}^{+\infty}|f(x)|^{p} d x \leq B \sum_{n=-\infty}^{+\infty}|f(m)|^{p} .
$$

As we recall, for $p>1$ this inequality holds for functions of type equal to $\pi$, provided it is known that the function lies in $E^{p}$. For $0<p \leq 1$, the inequality cannot hold in general for functions of type equal to $\pi$, even for functions belonging to $E^{p}$. For example, let

$$
g_{n}(z)=\frac{n \sin (\pi z)}{\pi z(z-n)}
$$


Now

$$
g_{n}=-\frac{\sin \pi x}{\pi x}+\frac{\sin \pi x}{\pi(x-n)},
$$

so that for $x$ between 0 and $n,\left|g_{n}(x)\right| \geq\left|\frac{\sin \pi x}{\pi x}\right|$. Consequently

$$
\int_{-\infty}^{\infty}\left|g_{n}(x)\right|^{p} d x \geq \int_{0}^{|n|}\left|\frac{\sin \pi x}{\pi x}\right|^{p} d x,
$$

so that $\left\|g_{n}\right\|_{p} \sim|n|^{\frac{1}{p}-1}$, for $\frac{1}{2}<p<1$ and $\sim \log |n|$ for $p=1$, even though $\left\|\left\{g_{n}(k)\right\}\right\|_{l_{p}}=2^{\frac{1}{p}}$ for all $n$. Similar examples can be constructed for $0<p \leq \frac{1}{2}$. In particular, Plancherel and Pólya's inequality reveals a way to test if a function of type $\pi$ belongs to $E^{p}$; that is, we need only determine whether the samples $\left\{f\left(\frac{n}{2}\right)\right\}$ belong to $l_{p}$, due to the fact that $f\left(\frac{z}{2}\right)$ is of type $\frac{\pi}{2}$. It is this simple observation that allows us to show the connection between $E^{p}$ and $H^{p}(\mathbf{Z})$.

Theorem 6. Let $0<p \leq 1$. If $f$ belongs to $E^{p}$, then $\left\{(-1)^{n} f(n)\right\}$ belongs to $H^{p}(\mathbf{Z})$. Conversely, if $\left\{\alpha_{n}\right\}$ belongs to $H^{p}(\mathbf{Z})$, there is a unique $f \in E^{p}$ such that $f(n)=(-1)^{n} \alpha_{n}$.

Proof. Let $f \in E^{p}$. $f$ has a cardinal series representation

$$
f(z)=\sum_{n=-\infty}^{\infty} f(n) \operatorname{sinc}(z-n) .
$$

(Since $f$ is also in $E^{2}$, the cardinal series converges uniformly on compact subsets of C.)

From Theorem $4(\mathrm{i}),\left\{f\left(\frac{n}{2}\right)\right\} \in l_{p}$. For even $n$, we simply recover the original samples of $f$, which thus belong to $l_{p}$. For odd $n, n=2 k+1, k \in \mathbf{Z}$,

$$
\begin{aligned}
f\left(\frac{2 k+1}{2}\right) & =\sum_{m} f(m) \operatorname{sinc}\left(\frac{2 k+1}{2}-m\right)=\sum_{m} f(m) \frac{\sin \pi\left(\frac{2 k+1}{2}-m\right)}{\pi\left(\frac{2 k+1}{2}-m\right)} \\
& =\frac{\sin \left(\frac{2 k+1}{2}\right) \pi}{\pi} \sum_{m} \cos (m \pi) f(m) \frac{1}{k-m+\frac{1}{2}} \\
& =\frac{(-1)^{k}}{\pi} \sum_{m} \frac{(-1)^{m} f(m)}{k-m+\frac{1}{2}}=\frac{(-1)^{k}}{\pi} H\left(\left\{(-1)^{m} f(m)\right\}\right)(k) .
\end{aligned}
$$

Since $\left\{f\left(\frac{2 k+1}{2}\right)\right\} \in l_{p}$, it follows that the Hilbert transform of $\left\{\left(-1^{n}\right) f(n)\right\} \in l_{p}$, whereby $\left\{(-1)^{n} f(n)\right\} \in H^{p}(\mathbf{Z})$.

Next suppose $\left\{\alpha_{n}\right\} \in H^{p}(\mathbf{Z})$. We form the cardinal series

$$
g(z)=\sum_{n=-\infty}^{\infty}(-1)^{n} \alpha_{n} \operatorname{sinc}(z-n) .
$$

Since $\left\{\alpha_{n}\right\} \in l_{p}, g$ is at least in $E^{2}$, and thus we know that the cardinal series converges uniformly on compacta. Now $g\left(\frac{z}{2}\right)$ is of type $\frac{\pi}{2}$; thus we may apply Theorem 4(i). The above calculations with the cardinal series show that the sequence of samples of $g\left(\frac{z}{2}\right)$ at the even integers is $\left\{\alpha_{k}\right\}$, and the sequence of samples of $g\left(\frac{z}{2}\right)$ at the odd integers is $\left\{\frac{(-1)^{k}}{\pi} H\left(\left\{\alpha_{n}\right\}\right)(k)\right\}_{k \in \mathbf{Z}}$. Consequently the sequence $\left\{g\left(\frac{n}{2}\right)\right\}$ belong to $l_{p}$, whereby $g \in E^{p}$. 
This proof shows that we can map $E^{p}$ onto $H^{p}(\mathbf{Z})$ via the map $f \rightarrow$ $\left\{(-1)^{n} f(n)\right\}$. For a function $f$ in $E^{p}$,

$$
\begin{aligned}
\sum_{n=-\infty}^{\infty}\left|f\left(\frac{n}{2}\right)\right|^{p} & =\sum_{k=-\infty}^{\infty}|f(k)|^{p}+\sum_{k=-\infty}^{\infty}\left|f\left(\frac{2 k+1}{2}\right)\right|^{p} \\
& =\sum_{k=-\infty}^{\infty}|f(k)|^{p}+\frac{1}{\pi} \sum_{k=-\infty}^{\infty}\left|\sum_{m=-\infty}^{\infty} \frac{(-1)^{m} f(m)}{k-m+\frac{1}{2}}\right|^{p} .
\end{aligned}
$$

It follows from Theorem 4 and the above proof that there are constants $C_{1}, C_{2}>0$ so that

$$
C_{1}\left\|\left\{(-1)^{n} f(n)\right\}\right\|_{H^{p}} \leq\|f\|_{p} \leq C_{2}\left\|\left\{(-1)^{n} f(n)\right\}\right\|_{H^{p}}
$$

for all $f \in E^{p}$.

Thus we see that the $H^{p}(\mathbf{Z})$ quasinorm is equivalent to the $E^{p}$ quasinorm, so that the map from $E^{p}$ onto $H^{p}(\mathbf{Z})$ is continuous, thereby yielding the following result.

Theorem 7. For $0<p \leq 1, E^{p}$ is isomorphic to $H^{p}(\mathbf{Z})$.

\section{Comments}

It is clear that sequences in $H^{p}(\mathbf{Z}), 0<p \leq 1$, must sum to zero. Consequently, for a function in $E^{p}, \sum_{n \in \mathbf{Z}}(-1)^{n} f(n)=0$; this also follows from well-known facts from classical harmonic analysis. For a function $f$ in $E^{p}$, the Fourier transform $\hat{f}$ is continuous on $\mathbf{R}$ and 0 off of $[-\pi, \pi]$. The above summation simply reflects the fact that $\hat{f}( \pm \pi)=0$. In fact, it is the cancellation that distinguishes $H^{p}(\mathbf{Z})$ from $l_{p}$ and, consequently, essentially what distinguishes $E^{p}$ from $L_{p}$, for $0<p \leq 1$. As for $H^{p}(\mathbf{Z})$, membership in $E^{p}$ requires progressively greater cancellation (actually, oscillation) for progressively smaller values of $p$. We will further examine this and other properties of $E^{p}$ in a subsequent paper [3].

\section{ACKNOWLEDGMENTS}

I thank Professor Nigel Kalton for some helpful suggestions regarding this paper. I also thank Professor Joel Shapiro, whose question initially prompted my investigations of the Paley-Wiener spaces.

\section{REFERENCES}

1. R. P. Boas, Entire functions, Academic Press, New York, 1954.

2. R. R. Coifman and G. Weiss, Extensions of Hardy spaces and their use in analysis, Bull. Amer. Math. Soc. 83 (1977), 569-645.

3. C. M. Eoff, The linear topological properties of the Paley-Wiener spaces, $E^{p}, 0<p \leq 1$, (in preparation).

4. J. B. Garnett, Bounded analytic functions, Academic Press, New York, 1981.

5. J. R. Higgins, Five short stories about the cardinal series, Bull. Amer. Math. Soc. (N.S.) 12 (1985), 45-89. 
6. M. Plancherel and G. Pólya, Fonctions entières et integrales de Fourier multiples, Comment. Math. Helv. 10 (1937), 110-163.

7. R. Rochberg, Toeplitz and Hankel operators on the Paley-Wiener space, Integral Equations Operator Theory 10 (1987), 187-235.

Department of Mathematical Sciences, University of ARKansas, Fayetteville, ARKANSAS 72701

E-mail address: ce24958@uaf sysb 